PROCEEDINGS OF THE

AMERICAN MATHEMATICAL SOCIETY

Volume 133, Number 2, Pages 501-510

S 0002-9939(04)07595-1

Article electronically published on September 8, 2004

\title{
CONVERGENCE PROPERTIES OF MINIMAL VECTORS FOR NORMAL OPERATORS AND WEIGHTED SHIFTS
}

\author{
ISABELLE CHALENDAR AND JONATHAN R. PARTINGTON
}

(Communicated by N. Tomczak-Jaegermann)

\begin{abstract}
We study the behaviour of the sequence of minimal vectors corresponding to certain classes of operators on reflexive $L^{p}$ spaces, including multiplication operators and bilateral weighted shifts. The results proved are based on explicit formulae for the minimal vectors, and provide extensions of results due to Ansari and Enflo, and also Wiesner. In many cases the convergence of sequences associated with the minimal vectors leads to the construction of hyperinvariant subspaces for cyclic operators.
\end{abstract}

\section{INTRODUCTION}

The construction of minimal vectors $\left(y_{n}\right)$, corresponding to an operator $T$ on a Hilbert space, was introduced by Enflo and his collaborators [2, 7, 8 as a method of constructing hyperinvariant subspaces for certain classes of linear operators. Further work in this area, extending the concept to more general Banach spaces, may be found in [1, 4, 5, 14, 16, 17]. As a result of this work, it has become apparent that it is important to be able to determine when the sequence $\left(T^{n} y_{n}\right)$ converges, since in many cases this gives an explicit construction of hyperinvariant subspaces.

In this paper we begin by considering the convergence in the case that $T$ is a multiplication operator on a reflexive $L^{p}(\mu)$ space, providing an alternative proof, as well as a generalization, of certain results in [2] concerning normal operators, as well as an extension of the convergence results in [17. We make use of certain very explicit formulae for the minimal vectors that are available in the $L^{p}$ case, and which were first given in [5. Another class of operators that can be fully analysed is the class of weighted bilateral shift operators on a reflexive space $\ell^{p}(\mathbb{Z})$, and again we provide fairly complete convergence results (in particular, we are thereby able to give criteria for convergence for a large class of hyponormal operators). Finally, we make some observations about the case $\ell^{1}(\mathbb{Z})$, where the minimal vectors still exist; they are not necessarily unique, but can be found using linear programming techniques.

We shall employ the following conventions and notation.

All the operators considered in this paper will be bounded linear operators defined on (real or complex) Banach spaces. For such an operator $T$, we denote by

Received by the editors July 23, 2003 and, in revised form, October 16, 2003.

2000 Mathematics Subject Classification. Primary 41A29, 47A15, 47A16, 47B20.

Key words and phrases. Minimal vectors, hyperinvariant subspaces, multiplication operators, weighted shifts, hyponormal operators. 
$\sigma(T)$ the spectrum of $T$ and $\rho(T)$ its spectral radius. A nontrivial hyperinvariant subspace of an operator $T$ acting on a Banach space $\mathcal{X}$ is a closed subspace $\mathcal{M}$ such that $\{0\} \neq \mathcal{M} \neq \mathcal{X}$ and $A \mathcal{M} \subseteq \mathcal{M}$ for all $A \in\{T\}^{\prime}:=\{A: \mathcal{X} \rightarrow \mathcal{X}: A T=T A\}$. For an operator $T$ defined on a space $\mathcal{X}$, we say that a vector $x \in \mathcal{X}$ is cyclic, if the closed linear span of $\left\{x, T x, T^{2} x, \ldots\right\}$ is the whole of $\mathcal{X}$. The vector $x$ is hypernoncyclic, if the closed linear span of the vectors $\left\{A x: A \in\{T\}^{\prime}\right\}$ is a proper subspace of $\mathcal{X}$. The operator $T$ is cyclic if it possesses a cyclic vector.

Recall that a hyponormal operator $T$ on a Hilbert space is one satisfying $T^{*} T-$ $T T^{*} \geq 0$. This class includes the class of subnormal operators (i.e., the restrictions of normal operators to closed invariant subspaces).

A Banach space $\mathcal{X}$ is said to be smooth, if for every vector $x \in \mathcal{X} \backslash\{0\}$ there is a unique linear functional $\nu(x)$ in $\mathcal{X}^{*}$ such that $\|\nu(x)\|=1$ and $\nu(x) x=\|x\|$. Furthermore, $\mathcal{X}$ is said to be strictly convex, if, whenever $x$ and $y$ are linearly independent vectors in $\mathcal{X}$ then we have $\|x+y\|<\|x\|+\|y\|$.

Suppose that $T$ is an operator on $\mathcal{X}$, with dense range, and that $f_{0} \in \mathcal{X} \backslash\{0\}$. Take $\epsilon$ such that $\left\|f_{0}\right\|>\epsilon>0$. For $n=1,2, \ldots$, a (backward) minimal vector $y_{n}$ associated with $T, n, f_{0}$ and $\epsilon$, is defined to be a vector of minimal norm such that $\left\|T^{n} y_{n}-f_{0}\right\| \leq \epsilon$. It is known that, if $\mathcal{X}$ is reflexive, then such minimal vectors exist and satisfy $\left\|T^{n} y_{n}-f_{0}\right\|=\epsilon$. If, in addition, $\mathcal{X}$ is strictly convex, then the minimal vectors are unique (see [5]).

\section{Normal operators on $L^{p}(\mu)$}

2.1. Normal operators on a Hilbert space. In 2, the following result was given.

Theorem A. Let $T$ be a normal operator with a cyclic vector and dense range $\mathcal{R}(T)$. Suppose that $f_{0} \notin \mathcal{R}(T)$. For any $\epsilon$ with $0<\epsilon<\left\|f_{0}\right\|$, let $\left(y_{n}\right)$ denote the corresponding sequence of backward minimal vectors. Then the sequence $\left(T^{n} y_{n}\right)$ converges in norm to a hypernoncyclic vector.

It seems, however, that the proof given in [2] is imprecise, since it relies on the following assertion, to which we shall give a counterexample: let $f \in L^{2}(\mu)$, where $\mu$ is a regular Borel measure with compact support. Then given $\epsilon>0$ there are $\delta>0$ and $0<\alpha<1$ such that if

$$
g(z)= \begin{cases}0 & \text { if }|z|<\delta \\ \alpha f(z) & \text { if }|z|=\delta \\ f(z) & \text { for }|z|>\delta\end{cases}
$$

then $\|f-g\|=\epsilon . \quad$ However, suppose that we take $\mu$ to be $\mu_{1}+\mu_{2}$, where $\mu_{1}$ is Lebesgue measure on $[0,1]$ and $\mu_{2}$ is a Dirac measure at $1 / 2$. Define $f \in L^{2}([0,1], \mu)$ by $f \equiv 1$, and take $\epsilon=1 / \sqrt{2}$. It is easy to verify that we must take $\delta=1 / 2$ and $\alpha=1$. Note that $\mu\{z:|z|=\delta\}>0$.

We now prove a slightly more general result than Theorem A, using only the spectral theorem for self-adjoint operators. It provides also a generalization of the main theorem of [17, which considers the case of a normal operator on a finitedimensional space.

Proposition 2.1. Let $T$ be a normal operator on a Hilbert space $\mathcal{H}$, with dense range. Suppose that $f_{0} \in \mathcal{H}$. For any $\epsilon$ with $0<\epsilon<\left\|f_{0}\right\|$, let $\left(y_{n}\right)$ denote the corresponding sequence of backward minimal vectors. Then the sequence $\left(T^{n} y_{n}\right)$ 
converges in norm. If, in addition, $T$ has a cyclic vector, then $\lim _{n \rightarrow \infty} T^{n} y_{n}$ is hypernoncyclic.

Proof. We shall make use of the expression

$$
f_{0}-T^{n} y_{n}=\left(I+\mu_{n} T^{n} T^{* n}\right)^{-1} f_{0},
$$

where $\mu_{n}$ is positive and uniquely determined by $\left\|f_{0}-T^{n} y_{n}\right\|=\epsilon$. We write $A$ for the positive self-adjoint operator $T T^{*}$. Thus

$$
g_{n}:=f_{0}-T^{n} y_{n}=\left(I+\mu_{n} A^{n}\right)^{-1} f_{0},
$$

since $T$ is normal. We now work entirely with the operator $A$, which we may represent by a unitarily equivalent operator, namely the operator $M_{\phi}$ on a space $L^{2}(X, \mu)$, given by pointwise multiplication by a non-negative function $\phi \in L^{\infty}(\mu)$ (see the spectral theorem, as given in [12]).

We observe that, under this identification,

$$
g_{n}(z)=\frac{f_{0}(z)}{1+\mu_{n} \phi(z)^{n}} \quad(z \in X) .
$$

Since $\left|g_{n}(z)\right| \leq\left|f_{0}(z)\right|$, it is sufficient to establish pointwise convergence almost everywhere, after which we obtain convergence in norm by the dominated convergence theorem. For $r \in[0, \rho(A)]$ we define

$$
L_{1}(r)=\limsup _{n \rightarrow \infty} \frac{1}{1+\mu_{n} r^{n}} \quad \text { and } \quad L_{2}(r)=\liminf _{n \rightarrow \infty} \frac{1}{1+\mu_{n} r^{n}} .
$$

Note that $0 \leq L_{2}(r) \leq L_{1}(r) \leq 1$ for all $r$ and that $L_{1}$ and $L_{2}$ are decreasing functions. If $L_{1}(r)>0$ for some value of $r$, then there is a sequence $\left(n_{k}\right)$ such that $\left(\mu_{n_{k}} r^{n_{k}}\right)$ remains bounded, so for $t<r$ we have $\mu_{n_{k}} t^{n_{k}} \rightarrow 0$, and hence $L_{1}(t)=1$ for $t<r$. We conclude immediately that if $L_{1}(s)<1$ for some $s$, then $L_{1}(t)=0$ for all $t>s$ (if not, we would have $L_{1}(s)=1$ ).

Thus there is a number $r_{1} \in[0, \rho(A)]$ such that

$$
L_{1}(t)= \begin{cases}1 & \text { for } t<r_{1}, \\ 0 & \text { for } t>r_{1},\end{cases}
$$

and similarly we have a number $r_{2} \in[0, \rho(A)]$ with $r_{2} \leq r_{1}$ such that

$$
L_{2}(t)= \begin{cases}1 & \text { for } t<r_{2} \\ 0 & \text { for } t>r_{2}\end{cases}
$$

Now we have

$$
\epsilon^{2}=\limsup _{n \rightarrow \infty}\left\|g_{n}\right\|^{2} \geq \int_{0 \leq \phi(z)<r_{1}}\left|f_{0}(z)\right|^{2} d \mu(z)+\int_{\phi(z)=r_{1}} L_{1}\left(r_{1}\right)^{2}\left|f_{0}(z)\right|^{2} d \mu(z)
$$

and

$$
\epsilon^{2}=\liminf _{n \rightarrow \infty}\left\|g_{n}\right\|^{2} \leq \int_{0 \leq \phi(z)<r_{2}}\left|f_{0}(z)\right|^{2} d \mu(z)+\int_{\phi(z)=r_{2}} L_{2}\left(r_{2}\right)^{2}\left|f_{0}(z)\right|^{2} d \mu(z) .
$$


If $r_{1}=r_{2}$, then either $L_{1}\left(r_{1}\right)=L_{2}\left(r_{2}\right)$ or $f_{0}(z)=0$ a.e. on the set on which $\phi(z)=r_{1}$, and so the sequence $\left(g_{n}\right)$ converges almost everywhere. Otherwise,

$$
\begin{aligned}
\left(1-L_{2}\left(r_{2}\right)^{2}\right) \int_{\phi(z)=r_{2}}\left|f_{0}(z)\right|^{2} d \mu(z) & +\int_{r_{2}<\phi(z)<r_{1}}\left|f_{0}(z)\right|^{2} d \mu(z) \\
& +L_{1}\left(r_{1}\right)^{2} \int_{\phi(z)=r_{1}}\left|f_{0}(z)\right|^{2} d \mu(z) \leq 0
\end{aligned}
$$

and hence each term is zero. It follows that $f_{0}(z)=0$ almost everywhere on the set on which $L_{1}(r) \neq L_{2}(r)$. Hence $\left(g_{n}\right)$ converges in norm.

If $T$ has a cyclic vector, then by the spectral theorem we may take $X=\sigma(A)$ and $\phi(z)=z$. Then $T^{n} y_{n}$ tends to a function $h$ (and necessarily $h \neq 0$ and $h \neq f_{0}$ ), with

$$
h(z)= \begin{cases}0 & \text { if } \phi(z)<r_{2}, \\ f_{0}(z) & \text { if } \phi(z)>r_{1} .\end{cases}
$$

Moreover, $h L^{\infty}(X, \mu) \subseteq \chi_{\left[r_{2}, \infty\right)} L^{\infty}(X, \mu)$. The fact that $h$ is hypernoncyclic now follows immediately from Lemma 2.1 below, which is well known, but for which we add a proof for the sake of completeness.

Lemma 2.1. Let $1 \leq p<\infty$, let $T=M_{\phi}$ be a multiplication operator on $L^{p}(X, \mu)$ and suppose that $T$ is cyclic. Then the commutant of $T$ is $\left\{M_{\psi}: \psi \in L^{\infty}(X, \mu)\right\}$.

Proof. Let $f$ be a cyclic vector for $T$; clearly $f \neq 0$ a.e. Suppose that $A T=T A$, then $A T^{n} f=T^{n} A f$ for all positive integers $n$, and thus $A\left(T^{n} f\right)=\psi \cdot\left(T^{n} f\right)$, where $\psi$ denotes $A f / f$. Since $A$ is bounded and $A=M_{\psi}$ on a dense subset of $L^{p}(X, \mu)$, it follows that $\psi \in L^{\infty}(X, \mu)$, and $A=M_{\psi}$ everywhere.

It would be of interest to derive similar results for general subnormal operators on a Hilbert space. In the case when $T$ is subnormal and cyclic, a result of J. Bram and I. M. Singer (see [6, Cor. 5.3]) states that $T$ is unitarily equivalent to the operator $M_{z}$ on a space $P^{2}(\mu) \subseteq L^{2}(\mu)$ given as the closed linear span of the polynomials in $L^{2}(\mu)$, where $\mu$ is a compactly-supported measure on the plane. An example, analysed in [4, 14, is that of multiplication on the Hardy space $H^{2}$ by a rational outer function, which is easily seen to correspond to a measure supported on a curve in $\mathbb{C}$. A formula which was useful in 4 is the following:

$$
T^{n} y_{n}=\mu_{n} T^{n}\left(I+\mu_{n} T^{* n} T^{n}\right)^{-1} T^{* n} f_{0},
$$

and in this case $T^{*}=P M_{\bar{z}}$, where $P: L^{2}(\mu) \rightarrow P^{2}(\mu)$ is the standard orthogonal projection. In [4], the operator $A:=I+\mu_{n} T^{* n} T^{n}=P M_{\left(1+\mu_{n}|z|^{2 n}\right)}$ is a Toeplitz operator, which could be inverted using a spectral factorization; however, this technique does not appear to be available in general. A full understanding of subnormal operators would require a full classification of the spaces $P^{2}(\mu)$, and this is not yet available (see [6]). Even the case of the multiplication operator by the function $z-1$ on the Bergman space appears to pose difficulties, since it would require an explicit formula for $A^{-1}$. Nevertheless, in Section 3 we shall analyse the particular case of weighted shift operators, including all hyponormal weighted shifts. These include some examples of multiplication operators on Hardy spaces of multiplyconnected domains, such as the annulus $\{z \in \mathbb{C}: s<|z|<1\}$ for $0<s<1$ (cf. [3, 11, 13, 18]). 
2.2. Multiplication operators on $L^{p}$ spaces. Let us now generalize the results of the previous section to spaces $L^{p}(X, \mu)$ for $1<p<\infty$. By the results of [5] Thm. 2.1], we know that minimal vectors $y_{n}$ still exist, and can be characterized by the formula

$$
\left(T^{n}\right)^{*} \nu\left(f_{0}-T^{n} y_{n}\right)=\mu_{n} \nu\left(y_{n}\right),
$$

for some $\mu_{n}>0$, where $\nu: L^{p}(X, \mu) \backslash\{0\} \rightarrow L^{q}(X, \mu)$ is the duality mapping, given by

$$
\nu(g)=\frac{\bar{g}|g|^{p-2}}{\|g\|_{p}^{p-1}}
$$

and, as usual, $1 / p+1 / q=1$. We now establish the norm convergence of the sequence $\left(T^{n} y_{n}\right)$ in the case when $T=M_{\phi}$ and $\phi \in L^{\infty}(X, \mu)$.

Theorem 2.1. Let $1<p<\infty$ and suppose that $T: L^{p}(X, \mu) \rightarrow L^{p}(X, \mu)$ is the operator $M_{\phi}$ of multiplication by $\phi \in L^{\infty}(X, \mu)$, and that it has dense range. Suppose that $f_{0} \in L^{p}(X, \mu)$. For any $\epsilon$ with $0<\epsilon<\left\|f_{0}\right\|$, let $\left(y_{n}\right)$ denote the corresponding sequence of backward minimal vectors. Then the sequence $\left(T^{n} y_{n}\right)$ converges in norm. If, in addition, $T$ is cyclic, then $T^{n} y_{n}$ converges to a hypernoncyclic vector.

Proof. Let us write $\beta_{n}=\left\|y_{n}\right\|_{p}$. By (3) and (4), we have

$$
\phi(z)^{n} \frac{\overline{\left(f_{0}-T^{n} y_{n}\right)(z)}\left|\left(f_{0}-T^{n} y_{n}\right)(z)\right|^{p-2}}{\epsilon^{p-1}}=\mu_{n} \frac{\overline{y_{n}(z)}\left|y_{n}(z)\right|^{p-2}}{\beta_{n}^{p-1}},
$$

because $T^{*}=M_{\phi}$ acting on $L^{q}(X, \mu)$, since the duality pairing is bilinear rather than sesquilinear. Since $y_{n}=\left(T^{n} y_{n}\right) / \phi^{n}$, it follows that

$$
\frac{\left(f_{0}-T^{n} y_{n}\right)(z)}{\left(T^{n} y_{n}\right)(z)}=\mu_{n} \frac{\epsilon^{p-1}}{\beta_{n}^{p-1}} \frac{1}{|\phi(z)|^{2 n}} \frac{\left|y_{n}(z)\right|^{p-2}}{\left|\left(f_{0}-T^{n} y_{n}\right)(z)\right|^{p-2}} .
$$

Taking absolute values, we obtain

$$
\frac{\left|\left(f_{0}-T^{n} y_{n}\right)(z)\right|^{p-1}}{\left|\left(T^{n} y_{n}\right)(z)\right|^{p-1}}=\mu_{n} \frac{\epsilon^{p-1}}{\beta_{n}^{p-1}} \frac{1}{|\phi(z)|^{n p}} .
$$

Write

$$
K_{n}(r)=\mu_{n}^{1 /(p-1)} \frac{\epsilon}{\beta_{n}} \frac{1}{r^{n p /(p-1)}}
$$

so that

$$
\frac{\left(f_{0}-T^{n} y_{n}\right)(z)}{\left(T^{n} y_{n}\right)(z)}=\frac{\left|\left(f_{0}-T^{n} y_{n}\right)(z)\right|}{\left|\left(T^{n} y_{n}\right)(z)\right|}=K_{n}(|\phi(z)|) .
$$

Thus,

$$
\left(f_{0}-T^{n} y_{n}\right)(z)=\frac{K_{n}(|\phi(z)|)}{1+K_{n}(|\phi(z)|)} f_{0}(z):=J_{n}(|\phi(z)|) f_{0}(z),
$$

say, where $0 \leq J_{n}(r) \leq 1$ for each $r \geq 0$.

As in the proof of Proposition 2.1, we shall establish pointwise convergence almost everywhere and use the dominated convergence theorem to deduce norm convergence. Accordingly, let $L_{1}(r)=\limsup _{n \rightarrow \infty} J_{n}(r)$ and $L_{2}(r)=\liminf _{n \rightarrow \infty} J_{n}(r)$. Then $0 \leq L_{1}(r) \leq 1$ for all $r$, and $L_{1}$ is a decreasing function of $r$; the same holds for $L_{2}(r)$, and clearly $L_{2}(r) \leq L_{1}(r)$. 
Exactly as in the proof of Proposition 2.1 there exist $r_{2} \leq r_{1}$ such that (11) and (21) hold. Write $g_{n}=f_{0}-T^{n} y_{n}$. Then we have

$$
\epsilon^{p}=\limsup _{n \rightarrow \infty}\left\|g_{n}\right\|^{p} \geq \int_{0 \leq|\phi(z)|<r_{1}}\left|f_{0}(z)\right|^{p} d \mu(z)+\int_{|\phi(z)|=r_{1}} L_{1}\left(r_{1}\right)^{p}\left|f_{0}(z)\right|^{p} d \mu(z)
$$

and

$$
\epsilon^{p}=\liminf _{n \rightarrow \infty}\left\|g_{n}\right\|^{p} \leq \int_{0 \leq|\phi(z)|<r_{2}}\left|f_{0}(z)\right|^{p} d \mu(z)+\int_{|\phi(z)|=r_{2}} L_{2}\left(r_{2}\right)^{p}\left|f_{0}(z)\right|^{p} d \mu(z) .
$$

If $r_{1}=r_{2}$, then either $L_{1}\left(r_{1}\right)=L_{2}\left(r_{2}\right)$ or $f_{0}(z)=0$ a.e. on the set on which $|\phi(z)|=r_{1}$, and so the sequence converges almost everywhere. Otherwise

$$
\begin{aligned}
\left(1-L_{2}\left(r_{2}\right)^{p}\right) \int_{|\phi(z)|=r_{2}}\left|f_{0}(z)\right|^{p} d \mu(z) & +\int_{r_{2}<|\phi(z)|<r_{1}}\left|f_{0}(z)\right|^{p} d \mu(z) \\
& +L_{1}\left(r_{1}\right)^{2} \int_{|\phi(z)|=r_{1}}\left|f_{0}(z)\right|^{p} d \mu(z) \leq 0,
\end{aligned}
$$

and hence each term is zero. It follows that $f_{0}(z)=0$ almost everywhere on the set on which $L_{1}(r) \neq L_{2}(r)$. Hence $\left(g_{n}\right)$ converges in norm.

If $T$ is cyclic, then the commutant of $M_{\phi}$ is the set $\left\{M_{\psi}: \psi \in L^{\infty}(X, \mu)\right\}$, by Lemma 2.1 and $h L^{\infty}(X, \mu) \subseteq \chi_{\left\{z:|\phi(z)| \geq r_{2}\right\}} L^{\infty}(X, \mu)$. Thus $h$ is hypernoncyclic.

\section{WeIGHTED SHIFT OPERATORS}

3.1. Weighted shifts on a Hilbert space. We now consider a class of operators which are not, in general, normal, but in which there are many hyponormal operators of interest. These are bilateral weighted shifts (note that unilateral weighted shifts do not have dense range, and will therefore not be considered here). by

Accordingly, let $T$ be a bilateral weighted shift on $\ell^{2}(\mathbb{Z})$ with dense range, defined

$$
T e_{k}=\alpha_{k} e_{k+1} \quad(k \in \mathbb{Z}),
$$

where as usual $\left(e_{k}\right)_{k \in \mathbb{Z}}$ denotes the standard basis of $\ell^{2}(\mathbb{Z})$. We shall assume that $\alpha_{k}>0$ for all $n$, and that $\sup _{k \in \mathbb{Z}} \alpha_{k}<\infty$ (hence $T$ is a bounded operator). Then clearly $T^{*} e_{k}=\alpha_{k-1} e_{k-1}$ for each $k \in \mathbb{Z}$.

As usual we take a vector $f_{0}$ and a positive constant $\epsilon<\left\|f_{0}\right\|$, and construct the backward minimal vectors $\left(y_{n}\right)$ corresponding to $T, y_{0}$ and $\epsilon$. Since

$$
f_{0}-T^{n} y_{n}=\left(I+\mu_{n} T^{n} T^{* n}\right)^{-1} f_{0},
$$

where $\mu_{n}$ is the unique positive constant such that $\left\|f_{0}-T^{n} y_{n}\right\|=\epsilon$, we see that, if $f_{0}=\sum_{k \in \mathbb{Z}} c_{k} e_{k}$, then

$$
f_{0}-T^{n} y_{n}=\sum_{k=-\infty}^{\infty} \frac{c_{k}}{1+\mu_{n}\left(\alpha_{k-1}^{2} \cdots \alpha_{k-n}^{2}\right)} e_{k}
$$

where $\mu_{n}$ satisfies

$$
\sum_{k=-\infty}^{\infty} \frac{\left|c_{k}\right|^{2}}{\left(1+\mu_{n}\left(\alpha_{k-1}^{2} \cdots \alpha_{k-n}^{2}\right)\right)^{2}}=\epsilon^{2} .
$$


By the dominated convergence theorem, $f_{0}-T^{n} y_{n}$ converges if and only if

$$
\lim _{n \rightarrow \infty} \frac{1}{1+\mu_{n}\left(\alpha_{k-1}^{2} \cdots \alpha_{k-n}^{2}\right)} \quad \text { exists for all } k \in \mathbb{Z} \text { with } c_{k} \neq 0 \text {. }
$$

Theorem 3.1. Let $\left(y_{n}\right) \subset \ell^{2}(\mathbb{Z})$ be the sequence of backward minimal vectors corresponding to $T, y_{0}$ and $\epsilon$. Suppose that $\lim _{m \rightarrow-\infty} \alpha_{m}$ exists. Then the sequence $\left(T^{n} y_{n}\right)$ converges. Conversely, if $\lim _{m \rightarrow-\infty} \alpha_{m}$ does not exist, then there is a finitely-supported vector $f_{0}$ for which $\left(T^{n} y_{n}\right)$ does not converge for any $\epsilon$ with $0<\epsilon<\left\|f_{0}\right\|$.

Proof. Under the hypothesis that $\lim _{m \rightarrow-\infty} \alpha_{m}$ exists, let $k$ be an index with $c_{k} \neq$ 0 . If

$$
\lim _{n \rightarrow \infty} \frac{1}{1+\mu_{n}\left(\alpha_{k-1}^{2} \cdots \alpha_{k-n}^{2}\right)}
$$

does not exist, then there exist $L_{1}>L_{2}$ and subsequences $\left(n_{j}\right)$ and $\left(m_{j}\right)$ such that

$$
\lim _{j \rightarrow \infty} \frac{1}{1+\mu_{n_{j}}\left(\alpha_{k-1}^{2} \cdots \alpha_{k-n_{j}}^{2}\right)}=L_{1}
$$

and

$$
\lim _{j \rightarrow \infty} \operatorname{frac} 11+\mu_{m_{j}}\left(\alpha_{k-1}^{2} \cdots \alpha_{k-m_{j}}^{2}\right)=L_{2} .
$$

Hence, for any $\ell \in \mathbb{Z}$, we have

$$
\lim _{j \rightarrow \infty} \frac{1}{1+\mu_{n_{j}}\left(\alpha_{\ell-1}^{2} \cdots \alpha_{\ell-n_{j}}^{2}\right)}>\lim _{j \rightarrow \infty} \frac{1}{1+\mu_{m_{j}}\left(\alpha_{\ell-1}^{2} \cdots \alpha_{\ell-m_{j}}^{2}\right)} .
$$

Now from the dominated convergence theorem, we obtain a contradiction, using (6).

Conversely, consider the extremal problem with $f_{0}=e_{0}+e_{1}$ and $0<\epsilon<\sqrt{2}$. We have

$$
\frac{1}{\left(1+\mu_{n}\left(\alpha_{-1}^{2} \cdots \alpha_{-n}^{2}\right)\right)^{2}}+\frac{1}{\left(1+\mu_{n}\left(\alpha_{0}^{2} \cdots \alpha_{1-n}^{2}\right)\right)^{2}}=\epsilon^{2},
$$

i.e.,

$$
\frac{1}{\left(1+\lambda_{n} \alpha_{-n}^{2}\right)^{2}}+\frac{1}{\left(1+\lambda_{n} \alpha_{0}^{2}\right)^{2}}=\epsilon^{2},
$$

where $\lambda_{n}=\mu_{n} \alpha_{-1}^{2} \cdots \alpha_{1-n}^{2}$. If $\lim _{m \rightarrow-\infty} \alpha_{m}$ does not exist, then (17) implies that the sequence $\left(\lambda_{n}\right)$ cannot converge, and thus the minimal vectors $\left(T^{n} y_{n}\right)$ do not converge.

Remark 3.1. Since $\left(T^{*} T-T T^{*}\right) e_{k}=\left(\alpha_{k}^{2}-\alpha_{k-1}^{2}\right) e_{k}$, it is clear that a weighted shift operator is hyponormal if and only if the sequence $\left(\alpha_{k}\right)$ is increasing (cf. [6. Prop. 6.6]). An obvious corollary of Theorem 3.1 is that for a hyponormal weighted shift the sequence $\left(T^{n} y_{n}\right)$ of minimal vectors always converges in norm. This result may be compared with Proposition 2.1 for normal operators; however, we cannot hope for a further result involving cyclic vectors, since any hyponormal bilateral weighted shift possessing cyclic vectors is necessarily normal (see [9, 10]). 
3.2. Weighted shifts on $\ell^{p}(\mathbb{Z})$. We now perform a similar convergence analysis in the case when $T$ is a bilateral weighted shift on $\ell^{p}(\mathbb{Z})$ for $1<p<\infty$. Here we need to use the expression (3) for backward minimal vectors, originally derived in [5]. We take a sequence of strictly positive weights $\left(\alpha_{k}\right)$ with $\sup _{k \in \mathbb{Z}} \alpha_{k}<\infty$, a nonzero vector $f_{0} \in \ell^{p}(\mathbb{Z})$ and an $\epsilon$ with $0<\epsilon<\left\|f_{0}\right\|$.

Theorem 3.2. Let $1<p<\infty$ and let $\left(y_{n}\right) \subset \ell^{p}(\mathbb{Z})$ be the sequence of backward minimal vectors corresponding to $T, y_{0}$ and $\epsilon$. Suppose that $\lim _{m \rightarrow-\infty} \alpha_{m}$ exists. Then the sequence $\left(T^{n} y_{n}\right)$ converges. Conversely, if $\lim _{m \rightarrow-\infty} \alpha_{m}$ does not exist, then there is a finitely-supported vector $f_{0}$ for which $\left(T^{n} y_{n}\right)$ does not converge for any $\epsilon$ with $0<\epsilon<\left\|f_{0}\right\|$.

Proof. We write $f_{0}=\sum_{k \in \mathbb{Z}} c_{k} e_{k}$, and suppose that

$$
f_{0}-T^{n} y_{n}=\sum_{k=-\infty}^{\infty} d_{k} e_{k}
$$

It is easily verified that we have

$$
y_{n}=\sum_{k=-\infty}^{\infty} \frac{c_{k}-d_{k}}{\alpha_{k-1} \cdots \alpha_{k-n}} e_{k-n},
$$

and so (3) gives

$$
\sum_{k=-\infty}^{\infty} \frac{\overline{d_{k}}\left|d_{k}\right|^{p-2}}{\epsilon^{p-1}}\left(\alpha_{k-1} \cdots \alpha_{k-n}\right) e_{k-n}=\mu_{n} \sum_{k=-\infty}^{\infty} \frac{\overline{c_{k}-d_{k}}\left|c_{k}-d_{k}\right|^{p-2}}{\left(\alpha_{k-1} \cdots \alpha_{k-n}\right)^{p-1} \beta_{n}^{p-1}} e_{k-n},
$$

where $\beta_{n}=\left\|y_{n}\right\|$. Hence, we see that for each $k \in \mathbb{Z}$, the ratio $d_{k} /\left(c_{k}-d_{k}\right)$ is non-negative, and it follows that

$$
\frac{d_{k}}{c_{k}-d_{k}}=\frac{1}{\lambda_{n}\left(\alpha_{k-1} \cdots \alpha_{k-n}\right)^{p /(p-1)}},
$$

where

$$
\lambda_{n}=\frac{\beta_{n}}{\epsilon \mu_{n}^{1 /(p-1)}}>0
$$

Thus

$$
f_{0}-T^{n} y_{n}=\sum_{k=-\infty}^{\infty} \frac{c_{k}}{1+\lambda_{n}\left(\alpha_{k-1} \cdots \alpha_{k-n}\right)^{p /(p-1)}} e_{k} .
$$

The remainder of the proof proceeds as in the proof of Theorem 3.1 by comparing expressions (5) and (8).

3.3. Remarks about weighted shifts on $\ell^{1}(\mathbb{Z})$. The space $\ell^{1}(\mathbb{Z})$ is neither reflexive nor strictly convex, and the methods of [5] no longer apply. However, since $\ell^{1}(\mathbb{Z})$ is a dual space, a weak* compactness argument shows the existence of minimal vectors $\left(y_{n}\right)$, and as in [5. Lem. 2.1], every minimal vector saturates the constraint, in the sense that $\left\|T^{n} y_{n}-f_{0}\right\|=\epsilon$.

Consider now the weighted shift $T$ on $\ell^{1}(\mathbb{Z})$, corresponding to a weight sequence $\left(\alpha_{k}\right)$. As usual, we suppose that $\alpha_{k}>0$ for each $k \in \mathbb{Z}$, and that $\sup _{k \in \mathbb{Z}} \alpha_{k}<$ 
$\infty$. Adopting the notation of the proof of Theorem [3.2, we have the following optimization problem:

$$
\min \sum_{k=-\infty}^{\infty} \frac{\left|c_{k}-d_{k}\right|}{\alpha_{k-1} \cdots \alpha_{k-n}} \quad \text { subject to } \sum_{k=-\infty}^{\infty}\left|d_{k}\right|=\epsilon
$$

Since

$$
\left\|\sum_{k=-\infty}^{\infty} b_{k} e_{k}\right\|_{1}=\left\|\sum_{k=-\infty}^{\infty}\left|b_{k}\right| e_{k}\right\|_{1},
$$

for all $\left(b_{k}\right) \in \ell^{1}(\mathbb{Z})$, it is easily seen that it is sufficient to be able to calculate minimal vectors in the case when $f_{0}=\sum_{k \in \mathbb{Z}} c_{k} e_{k}$ and each $c_{k}$ is non-negative. In this case we must have $0 \leq d_{k} \leq c_{k}$ for each $k \in \mathbb{Z}$, and thus it remains to solve the following linear programming problem:

$$
\max \sum_{k=-\infty}^{\infty} \frac{d_{k}}{\alpha_{k-1} \cdots \alpha_{k-n}} \quad \text { subject to } 0 \leq d_{k} \leq c_{k} \text { for each } k \quad \text { and } \quad \sum_{k=-\infty}^{\infty} d_{k}=\epsilon .
$$

We refer to [15] for basic information on linear programming. In this case, an inspection of the problem shows that we must have either $d_{k}=0$ or $d_{k}=c_{k}$ for all except at most one value of $k$, which depends on $n$.

If $\left(\alpha_{k}\right)$ is strictly increasing, then $\alpha_{k-1} \cdots \alpha_{k-n}$ increases strictly with $k$ for each $n$, and so there is an index $k_{n}$ such that $d_{k}=0$ for $k>k_{n}$ and $d_{k}=c_{k}$ for $k<k_{n}$. Since $\sum_{k=-\infty}^{\infty} d_{k}=\epsilon$, it follows that the sequence of vectors $\left(T^{n} y_{n}\right)$ is constant, thus trivially converges.

However, in general, even if supp $f_{0}$ is finite and $\lim _{k \rightarrow-\infty} \alpha_{k}$ exists, the $T^{n} y_{n}$ may not converge. To see this, take $f_{0}=e_{0}+e_{1}, \epsilon=1, \alpha_{m}=1$ for all $m \geq 0$ and $\alpha_{-m}=1+(-1)^{m} /(m+1)$ for $m=1,2, \ldots$. It is then easily verified that $T^{n} y_{n}$ takes the values $e_{0}$ and $e_{1}$ alternately, so the sequence does not converge.

It is also easy to construct examples when the minimal vectors are not unique: consider, for example, the case $f_{0}=e_{0}+e_{1}$ with $\epsilon=1$ and $\alpha_{k}=1$ for all $k$. Then $f_{0}-T^{n} y_{n}$ can take any value which is a convex combination of $e_{0}$ and $e_{1}$.

\section{ACKNOWLEDGEMEnts}

The authors are grateful to the European Research Training Network in Analysis and Operators for financial support. They also wish to thank the referee for reading the paper carefully and providing numerous helpful suggestions.

\section{REFERENCES}

[1] G. Androulakis, A note on the method of minimal vectors. Trends in Banach spaces and operator theory, Contemp. Math., 321:29-36, 2003.

[2] S. Ansari and P. Enflo. Extremal vectors and invariant subspaces. Trans. Amer. Math. Soc., 350:539-558, 1998. MR 98d:47019

[3] I. Chalendar and J. R. Partington. Approximation problems and representations of Hardy spaces in circular domains. Studia Math., 136(3):255-269, 1999. MR 2000i:30063

[4] I. Chalendar and J. R. Partington. Constrained approximation and invariant subspaces. J. Math. Anal. Appl., 280:176-187, 2003. MR 2004d:41027

[5] I. Chalendar, J. R. Partington, and M. Smith. Approximation in reflexive Banach spaces and applications to the invariant subspace problem. Proc. Amer. Math. Soc, 2003.

[6] J. B. Conway. The theory of subnormal operators, volume 36 of Mathematical Surveys and Monographs. American Mathematical Society, Providence, RI, 1991. MR 92h:47026 
[7] P. Enflo. Extremal vectors for a class of linear operators. In Functional analysis and economic theory (Samos, 1996), pages 61-64. Springer, Berlin, 1998. MR 2000k:47020

[8] P. Enflo and T. Hõim. Some results on extremal vectors and invariant subspaces. Proc. Amer. Math. Soc., 131(2):379-387, 2003. MR 2003i:47007

[9] R. Frankfurt. Subnormal weighted shifts and related function spaces. J. Math. Anal. Appl., 52(3):471-489, 1975. MR 58:2407

[10] D. A. Herrero. Eigenvectors and cyclic vectors for bilateral weighted shifts. Rev. Un. Mat. Argentina, 26:24-41, 1972/73. MR 49:1170

[11] D. Hitt. Invariant subspaces of $\mathcal{H}^{2}$ of an annulus. Pacific J. Math., 134(1):101-120, 1988. MR 90a:46059

[12] H. Radjavi and P. Rosenthal. Invariant subspaces. Springer-Verlag, New York, 1973. MR 51:3924

[13] D. Sarason. Nearly invariant subspaces of the backward shift. In Contributions to operator theory and its applications (Mesa, AZ, 1987), volume 35 of Oper. Theory Adv. Appl., pages 481-493. Birkhäuser, Basel, 1988. MR 90m:47012

[14] A. Spalsbury. Vectors of minimal norm. Proc. Amer. Math. Soc., 350:2737-2745, 1998. MR 98k:47009

[15] H. A. Taha. Operations research. Macmillan Co., New York, 1982. MR 88f:90057a

[16] V. G. Troitsky. Minimal vectors in arbitrary Banach spaces. Proc. Amer. Math. Soc., to appear.

[17] E. Wiesner. Backward minimal points for bounded linear operators on finite-dimensional vector spaces. Linear Algebra Appl., 338:251-259, 2001. MR 2002g:47033

[18] D. V. Yakubovich. Invariant subspaces of the operator of multiplication by $z$ in the space $E^{p}$ in a multiply connected domain. J. Soviet Math., 61(2):2046-2056, 1992. MR 91c:47061

Institut Girard Desargues, UFR de Mathématiques, Université Claude Bernard Lyon 1, 69622 Villeurbanne Cedex, France

E-mail address: chalenda@igd.univ-lyon1.fr

School of Mathematics, University of Leeds, Leeds LS2 9JT, United Kingdom

E-mail address: J.R.Partington@leeds.ac.uk 